\title{
DIE SIEDLUNGSLANDSCHAFT ZWISCHEN ANKARA UND DEM TUS GÖLÜ
}

\author{
Hartmut Scholz
}

Mit 6 Bildern

\begin{abstract}
Dic Naturlandschaft. Im Herzen des türkischen Hochlandes von Anatolien ruht in einem breiten, äußerlich jedoch nicht deutlich sichtbaren Binnenbecken der Tus Gölü. Im Gegensatz zum weiter ostwärts gelegenem Van-Gölü, der als größter türkischer Süßwassersee das Zentrum des zweiten Binnenbeckens einnimmt, ist der nahezu $80 \mathrm{~km}$ lange Tus Gölü mit $339 \%$, der salzhaltigste See der Erde. Gleich einem glitzernden Spiegel hebt er sich aus der Ferne ab, wobei die Ufer in der Luft zu schweben scheinen. Dabei gibt der Tus Gölü verschiedene Ausdrucksformen wider. Wo der See sich allmählich zurückgezogen hat, was insbesondere für die nordwestliche Region zutrifft, zeigt eine ausgesprochene Salzwüste den Übergang vom überkrusteten See zur Salzsteppe, die sich wiederum in respektabler Entfernung vom eigentlichen Ufer hält und mit ihrem schütteren Bewuchs lediglich den spärlichen Schafherden wie den frei herumlaufenden Eseln und Muli kümmerliche Nahrung bietet. Hier wirkt die Natur leblos, bis auf wenigen Luftwirbel, die der Steppenwind über die flachen Horizonte treibt, bis auf die staubig trockenen Schleier, welche den Straßenverkehr längs der schmalen Fahrbahn von Ankara nach Konya behindern.
\end{abstract}

Siedlungselemente. So ist es ganz verständlich, daß sich die Siedlungen um den Tus Gölü nur im gruppenhaften Beieinander finden. In einem Umkreis von rund $100 \mathrm{~km}$ des Tus Gölü, dessen Oberfläche sich mit dem Steppenstaub verschwistert hat, treten die Einzelsiedlungen vollends zurück. Nur kleine Hirtenhäuschen, notdürftig an die baumlosen Hänge geklebt, dienen den saisonalen Ansprüchen der Wanderwirtschaft. Lediglich im Norden des Gebietes um den Salzsee, nahe größerer Ansiedlungen, hält sich das armenische Bauernhaus.

Das armenische Bauernhaus. Seine Kennzeichen sind der quadratische, zweigeschossige Baukörper ohne irgend einen Anbau, wobei das Erdgeschoß als fensterloser Sockel steil aus dem Boden schießt und den Eindruck von etwas Festungsartigem vermittelt. Meistens ist diese Art der Gehöfte sehr alt. Man kann zudem sehr häufig beobachten, daß Tür und Fenster erst nachträglich eingefügt worden sind. Das Hausvieh wird im Erdgeschoß untergebracht, während das Obergeschoß - durch ein flachgeneigtes, schindelbedecktes Dach abgeschlossen der Wohnung vorbehalten bleibt. Der Zugang zur Wohnung ist oftmals nur über eine schmale Leiter möglich. Die Wohnung selbst setzt sich zumeist aus zwei großen oder vier kleineren Räumen zusammen. Teile des Obergeschosses läßt man gern überkragen, so daß der Sockel noch stärker herausgebildet wird und sich auch farblich von dem Obergeschoß abhebt. Das armenische

Abb. 1 Der Tus Gölü. So bietet sich der nördliche Tus Gölü dem Betrachter. Auf der polygonal aufgeteilten Salzkruste kann man bequem das andere Ufer erreichen, das von jeglicher Vegetation entblößt ist.

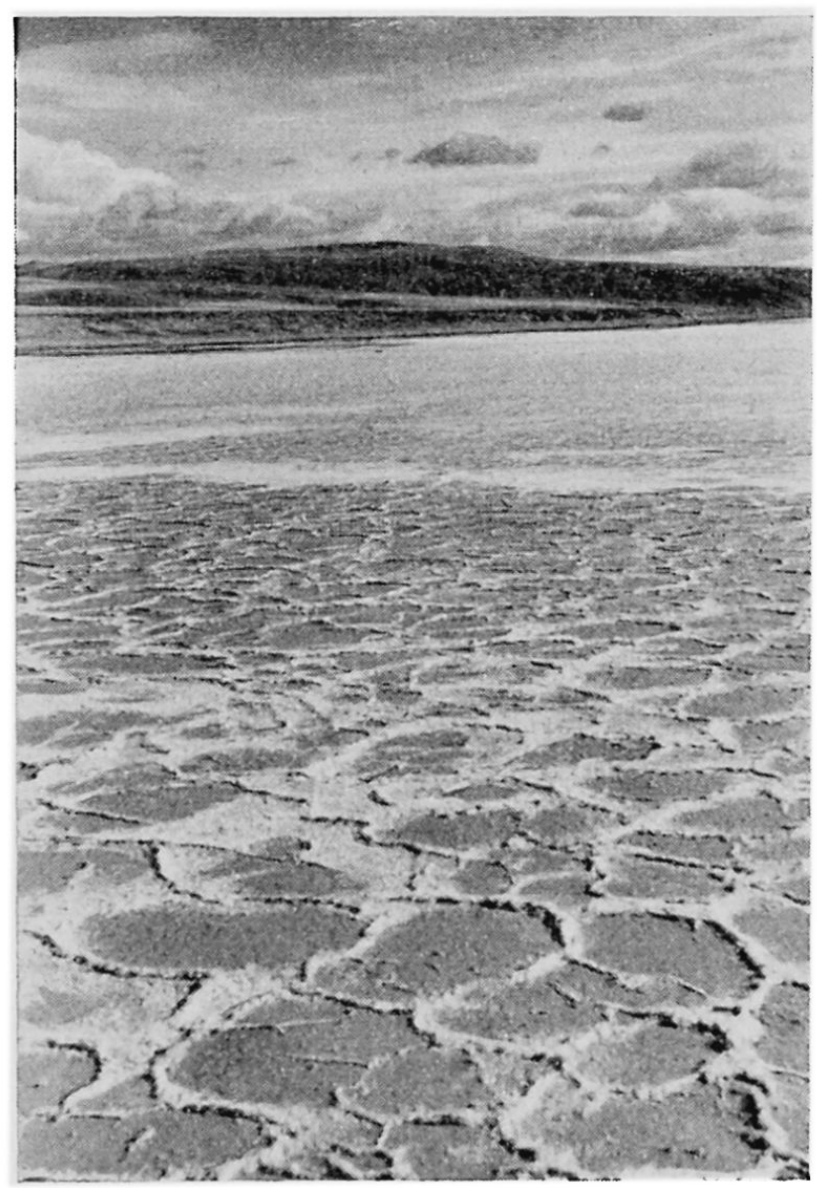




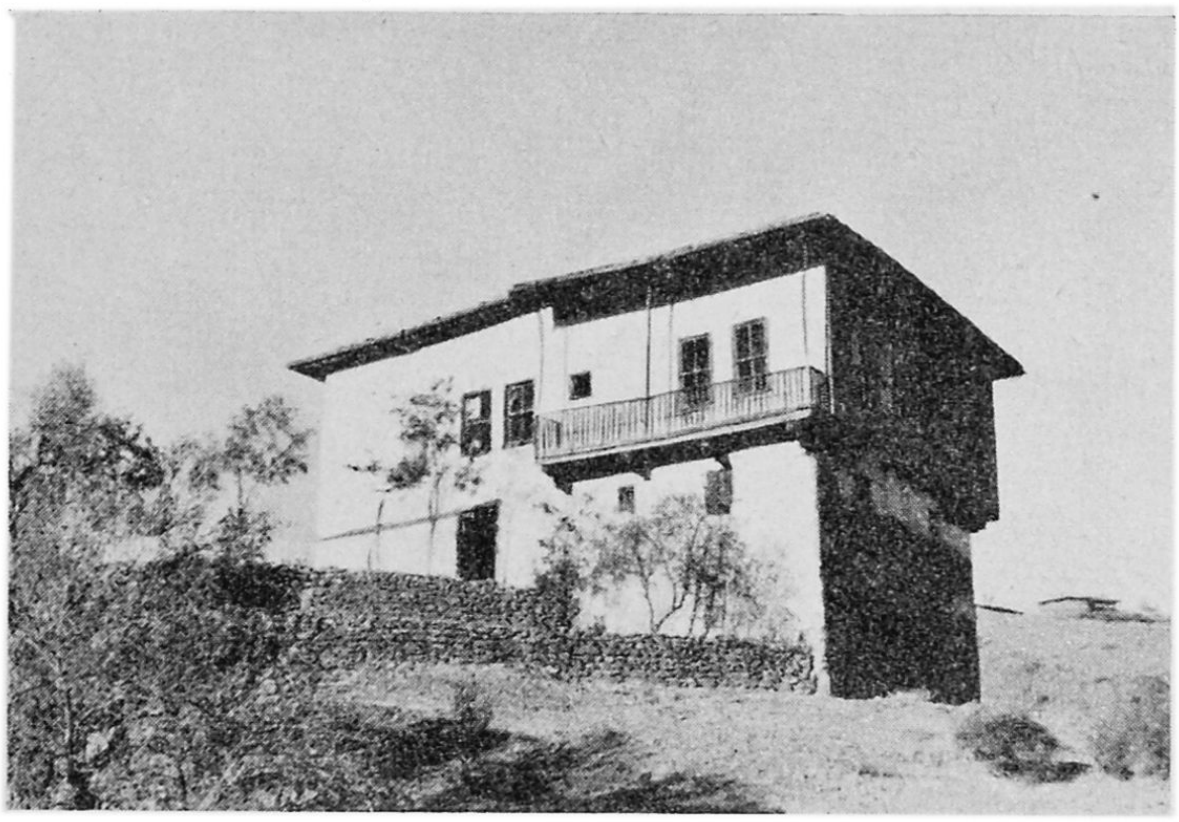

Abb. 2 Armenisches Bauernhaus als Relikt $10 \mathrm{~km}$ südostwärts des Stadtrandes von Ankara mit dem typischen Überkragen des Obergeschosses. Eine hausnahe Geröllmauer trennt den spärlichen Gemüsebau des (jartens ron der umliegenden Weinkultur mit teils künstlicher Bewässerung.

Bauerhaus hat sich als Funktion die landwirtschaftliche Tätigkeit bewahrt. In Stadtnähe und günstigen Hanganlagen sind die Besitzer in den letzten Jahrzehnten zu Weinbauern geworden.

Das anatolische Bauernhaus. Im Gegensatz zum armenischen Bauernhaus ist das Gegenstück des anatolischen Bauernhauses kaum alleinstehend $\mathrm{zu}$ finden. Zwischen dem Tus Gölü und der Landeshauptstadt Ankara, gruppieren sich die anatolischen Bauernhäuser in $20 \mathrm{~km}$ Abständen zu kleinen Dörfern und Weilern längs der gewichtigen Überlandstraße. Sie ist eine der bedeutendsten anatolischen Verkehrsverbindungen und ständig im Ausbau begriffen. Teils asphaltiert, teils nur mit einer festen Schotterdecke versehen, folgt sie der alten Linienführung des Karawanenweges, der noch im vorigen Jahrhundert die alten Handelsorte Konya und Ankara, das frühere Angora, verband.

Das anatolische Bauernhaus weist eine geringere Höhe als das armenische Bauernhaus auf. Auch hier sind Stall und Wohnung in einem allerdings mehr rechteckigen Baukörper untergebracht. Zumeist besteht der Hauptbaukörper aus zwei Teilen, die gegenseitig versetzt sind. Erstaunlich ist dabei immer wieder die Maßarbeit und Sym-

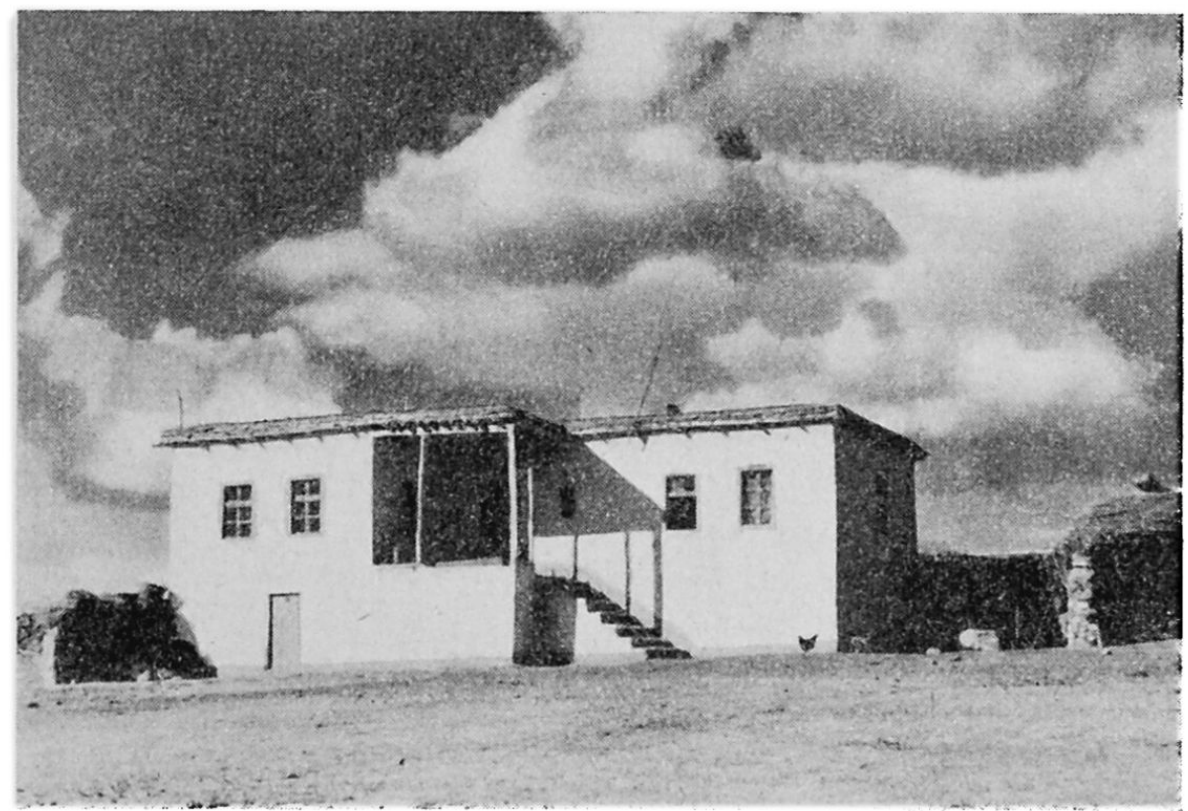

Abb. 3 Anatolisches Bauernhaus $30 \mathrm{~km}$ westlich des Tus Gölü als Charakteristikum der Hochsteppe. Bestechend die Sauberkeit und Symmetrie der Hausanordnung. 
Abb. 4 Hochlanddorf Bata in der vegetationslosen Landschaft zwischen dem Tus Gölü im Osten und dem Süßwassersee Gölbaschi im Westen. Die erdgeschossigen Lehmhäuser mit davorliegenden Dungballen ruhen auf der windabgekehrten Seite des Hügels.

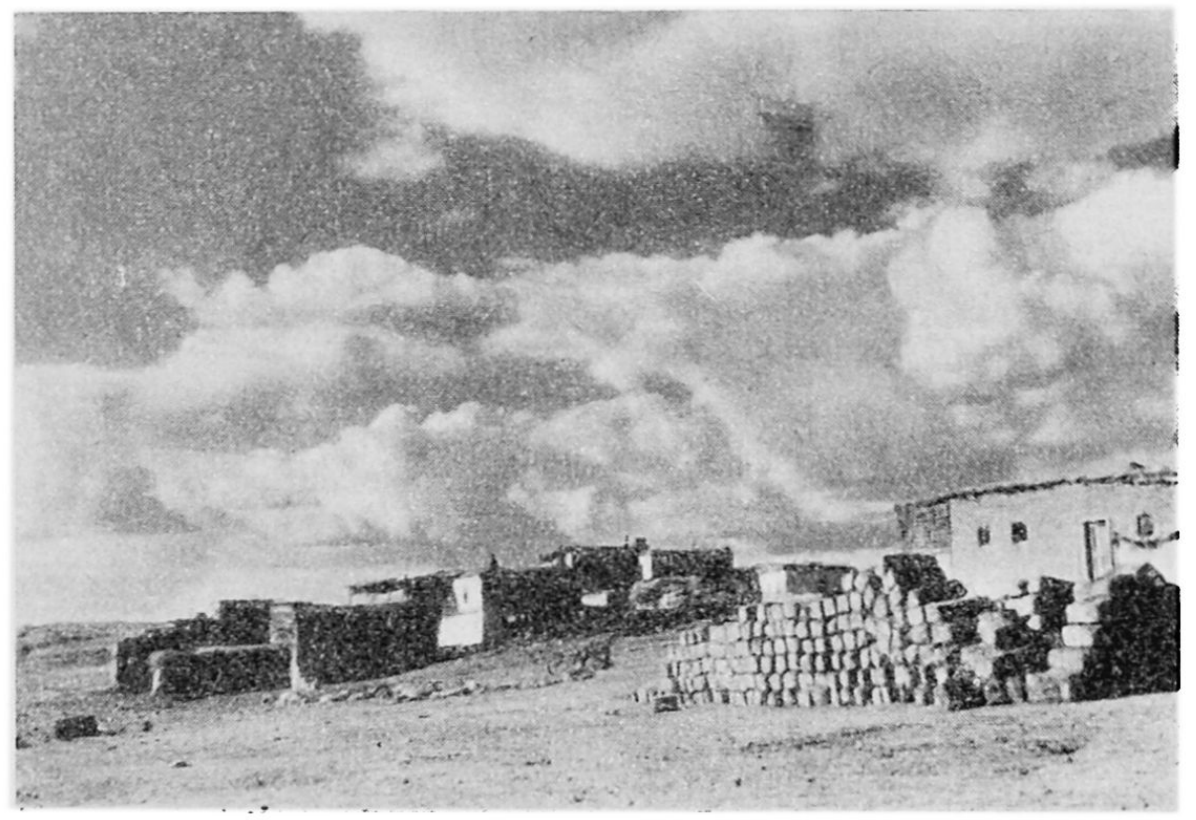

metrie des Bauwerkes, welches von den Bauherren selbst in mühevoller Kleinarbeit errichtet wird. Schmucke Veranden oder überdachte Vorräume - von einem Holzpfeiler getragen - und ebenso sauber angelegte Anbauten, künden von einer geschmackvollen Baukultur des ländlichen Siedlungswesens, die mit den Fachwerk- und Erkerbauten der alten Stadtkerne konkurrieren können.

Von den aus Luft getrocknetem Lehm errichteten anderen, überwiegend erdgeschossigen Häusern, die sich im gelb-oliven Äußeren über die Hügel ziehen, sind die anatolischen Gehöfte durchweg ziemlich streng getrennt. Manchmal bildet die Straße eine Grenze. Manchmal auch die Bachrinnsale, an denen verschiedentlich Zelte zu sehen sind, die erst weiter ostwärts, wo die Kurden den größeren Bevölkerungsanteil abgeben, bedeutend zunehmen. Auf der einen Seite der Straße liegen die anatolischen Gehöfte mit ihren großen Vorhöfen und Abstellfächen und auf der anderen Seite ruhen in gedrängter Folge die notdürftigen Lehmbauten, vor denen große getrocknete Dunghaufen aufgestapelt sind, um in erster Linie als Brennvorrat für den Hochlandwinter $z u$ dienen.

Abb. 5 Kurdische Bergsiedlung nordwestlich des Tus Gölü. Die Bauten bestehen aus breiten Lehmquadern, die den Wanderhirten im Winter als Unterkunft dienen.

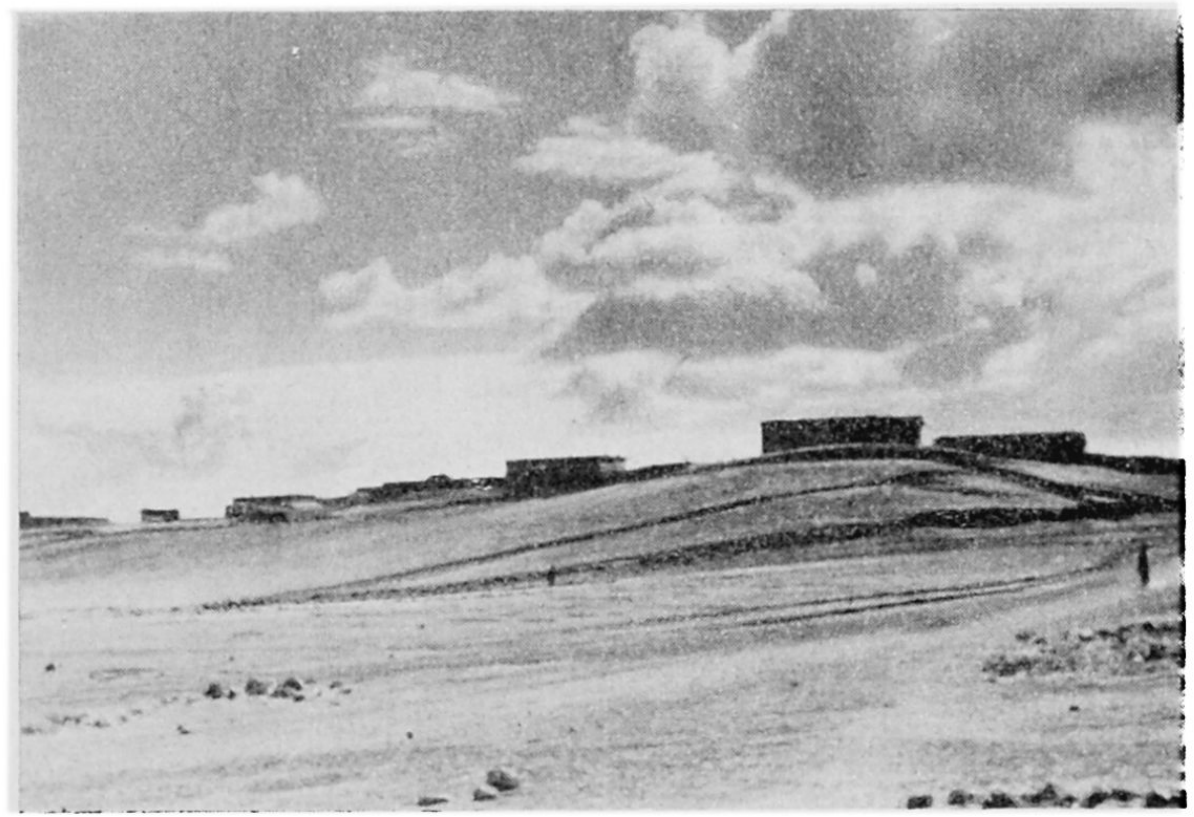




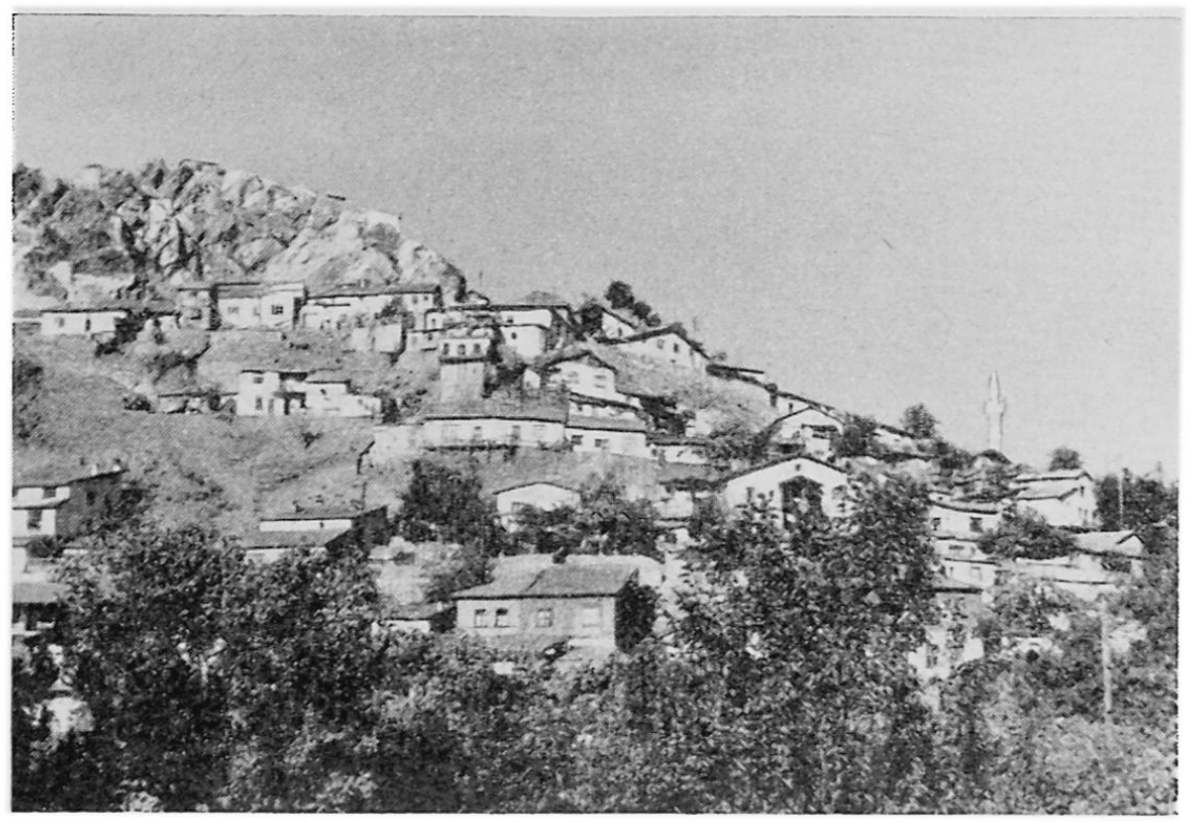

Abb. 6 aChampignon-

Häuser haben den Stadtrand von Ankara besetzt und 120000 Einwohner aufgenommen. Es sind bunte Gebilde, die auf Abbruch warten. In ihnen wohnen die vom Lande Geflohenen, um in der Stadt zu arbeiten.

Die Vorstadtsiedlung. Die seltsamsten Siedlungen Kleinasiens sind zweifellos diejenigen am Stadtrand von Ankara. Seitens der türkischen Ortsplanung werden sie treffend als «Champignons» bezeichnet, gewissermaßen «über Nacht gelandete Hütten». In buntem Auf und Ab, vom Rosenholz zum Violett, vom Ultramarin zum Seegrün, sind die schon fast nicht mehr fortzudenkenden Häuser bemalt. Ihre Entstehung ist wie folgt: Als Ankara 1923 von Kemal Atatürk zur Hauptstadt erkoren wurde, wuchs in der Steppe das Stadtwunder Anatoliens heran. Nicht zuletzt die gottähnliche Verehrung Atatürks, dessen Mausoleum auf dem Anit Kebir die Stadt überstrahlt und deren städtebauliche Dominante bildet, zog in unaufhörlicher Folge den Landmenschen aus der dürstenden Steppe in die vielversprechende Stadt. 1923 noch 35000 Einwohner zählend, die sich auf die wunderschöne Altstadt am Hang der alten Feste konzentrierten, stieg die Bevölkerung bis zum Sommer 1955 auf über 400000 Seelen an. Man kann sich diesen überquollenen Topf erst richtig vorstellen, wenn man bedenkt, daß die Stadt im Jahre 1928 vorausschauend auf 150000 Einwohner geplant wurde. Bereits Mitte der dreißiger Jahre waren diese Vorhaben erschöpft. So hängten sich an den mustergültigen Kern der Neustadt, der vom prächtigen Atatürk-Boulevard durchzogen wird, über alle Hügel des Südostens diese «Champignons». Es sind Elendsquartiere im wahrsten Sinne des Wortes. Ein ungeschriebenes Gesetz besagte, $\mathrm{da} \beta$ jeder bauen konnte, wenn zwischen Sonnenuntergang und Sonnenaufgang, also über Nacht, ein Dach auf vier Wänden gelegt werde, gleich, ob der Grund und Boden dem Staat oder der Stadt gehörte. So entstand diese Vorstadtsiedlung mit ihrem Gewirr von Wegen, für die die Versorgungseinrichtungen das größte Problem der sonst aufgeschlossenen Stadt darstellen. Nun sollen diese Mißbildungen einer nochmaligen weiträumigen Überbauung weichen, für die im Frühjahr 1955 die planerische Voraussetzung geschaffen wurde. Künstliche Bewässerung und eine systematische Baumanpflanzung stehen dabei Pate und haben bereits schon einen ansprechenden Grüngürtel um die Stadt gelegt und das Kleinod der Steppe gefestigt.

Siedlungsstruktur. Es dürfte nach Lage der Dinge kaum möglich sein, zwischen Ankara und dem Tus Gölü nach Regionen zu suchen, die von ganz bestimmten Siedlungselementen bestimmt werden. Wie das Gemisch der Bevölkerung aus den Strömungen der vergangenen Jahrhunderte schwierig $\mathrm{zu}$ analysieren ist, so steht es auch mit der Siedlung. Kurdenzelte, armenische und anatolische Gehöfte geben sich ein Stelldichein in einem breiten Grenzsaum, der lediglich den Gegensatz von Einzelund Gruppensiedlung schärfer herausstellt. Die Städte enden plötzlich, ohne Übergang. Die Einzelsiedlungen drängen sich in Stadtnähe, während auf dem weiten Hoch- 
land Weiler und Dörfer an der Tagesordnung sind, über viele Kilometer auf Sichtweite an alte Verkehrswege geheftet und vom spärlichen Bewuchs der Steppe lebend, auf denen die Schaf- und Ziegenherden ihre Wege ziehen. Mit dem Ausbau der Straßen wachsen auch moderne Stützpunkte heran, Tankstellen und Kaufläden, womit zugleich die einseitig ausgerichtete landwirtschaftliche Tätigkeit der Bevölkerung eine Änderung erfährt.

\section{L'HABITAT ENTRE ANKARA ET TUS GÖLÜ}

Entre Ankara et Tus Gölü s'etend une région aride qui présente une forme d'habitation qui lui est propre. A côté des huttes de bergers primitives, prédominent les fermes arméniennes et anatoliennes, qui déterminent l'aspect de la région. De plus, à la suite du rapide développement de la capitale Ankara, il s'est formé un curieux paysage suburbain composé de maisons sorties de terre comme des champignons. Ces quartiers misérables forment un contraste frappant avec l'aspect citadin moderne de la métropole; cependant, ils reculent déjà par l'application de mesures hygiéniques. En résumé, le paysage est ici, comme souvent ailleurs en Anatolie, l'expression de la diversité des peuples qui caractérise tout l'Orient.

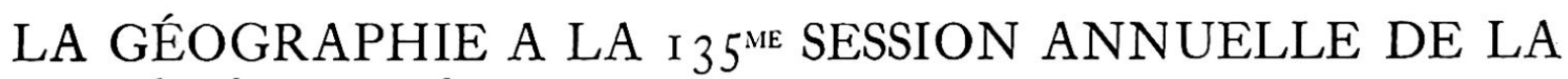 SOCIÉTÉ HELVÉTIQUE DES SCIENCES NATURELLES I 955}

\section{JEAN LINIGER}

La F.S.S.G. peut se réjouir des journées de Porrentruy à l'occasion de la $135^{\text {me }}$ session annuelle de la Société helvétique des sciences naturelles, les 24, 25 et 26 septembre 1955. La géographie a été à l'honneur avec la conférence présentée samedi soir 24 septembre par M. le professeur ARnold Heim sur l'Expédition suisse de 1954 1955 en Afrique centrale. La séance scientifique de la F.S.S.G. qui s'est déroulée dimanche 25 septembre de 8 h. à 12 h. à l'Ecole cantonale a été caractérisée par la quantité et la qualité remarquables des exposés ainsi que par la nombreuse affluence du public.

La participation scientifique de la Suisse française a été particulièrement importante. Elle est tout à l'honneur de la Société genevoise de géographie qui n'a pas envoyé moins de quatre conférenciers avec cinq exposés. La participation suisse-allemande a été également fort intéressante avec quatre exposés. Les conférences ont porté sur des questions de brûlante actualité comme le problème de la commune, du tunnel du Mont-Blanc et de la situation de la Suisse dans l'ère atomique, sur des problèmes de culture comme celui des minorités ou celui des rapports entre la topographie, l'ethnologie et l'histoire, sur des questions techniques comme l'atlas diacolor et la topographie de l'Himalaya, sur l'état présent de la science géographique en Allemagne, enfin sur la région de l'Ajoie. La plupart des exposés ont suscité des discussions qui ont témoigné de l'intérêt des sujets traités ainsi que de la valeur de leur présentation.

Samedi 24 septembre s'est tenue de $17 \mathrm{~h}$. à $18 \mathrm{~h}$. 15 une assemblée des délégués de la F.S.S. G. L'assemblée a pris connaissance avec regret de la démission de M. le professeur JeAn Gabus pour cause de maladie. La gratitude de la Fédération lui est acquise pour sa trop courte présidence et des vœux chaleureux sont formés pour sa prompte guérison. M. le $\mathrm{D}^{\mathrm{r}}$ JEAN LINIGER, vice-président en charge, est appelé à le remplacer. La section de Neuchâtel désignera un troisième membre pour compléter le comité central. L'assemblée a désigné à l'unanimité $\mathrm{M}$. le $\mathrm{D}^{\mathrm{r}}$ ANNaheim, ancien président central, pour représenter la Suisse au congrès de Rio de Janeiro. Cette candidature sera proposée au Conseil fédéral par le canal de la présidence de la Société helvétique des sciences naturelles. Une adresse sera envoyée à la Société nationale de géographie des Etats-Unis à l'occasion de la remise d'une médaille à son président, le $D^{r}$ La Gorce, pour ses 50 ans d'activité au service de la géographie. Des suggestions ont encore été faites pour le développement de l'activité scientifique par l'institution d'une journée consacrée à un thème commun ainsi que pour une meilleure information sur l'activité des diverses sociétés de géographie suisses et étrangères à tous les membres de chacune des sociétés membres de la F.S.S. G.

Les excursions, excellemment dirigées par M. ERzinger et NERTZ, dans le Doubs et dans le Jura, ont eu le plus grand succès. En dehors des assemblées administratives et scientifiques, 